А. П. Филимонова, Т. А. Юрьева. Априорные оценки градиента решения уравнения некоторого класса Монжа - Ампера

УДК 517.953

DOI: 10.18101/2304-5728-2019-1-49-55

\title{
АПРИОРНЫЕ ОЦЕНКИ ГРАДИЕНТА РЕШЕНИЯ УРАВНЕНИЯ НЕКОТОРОГО КЛАССА МОНЖА - АМПЕРА
}

\author{
(C) Филимонова Анна Павловна \\ кандидат физико-математических наук, доцент, \\ Амурский государственный университет \\ Россия, 675000, г. Благовещенск, ул. Игнатьевское шоссе, 21 \\ E-mail: ap.filimonova@mail.ru
}

\author{
(C) Юрьева Татьяна Александровна \\ кандидат педагогических наук, доцент, \\ Амурский государственный университет \\ Россия, 675000, г. Благовещенск, ул. Игнатьевское шоссе, 21 \\ E-mail: yuryevatat@mail.ru
}

Решение вопроса о существовании и единственности поверхностей с заданными геометрическими характеристиками в различных пространствах связано с отысканием априорных оценок решения в соответствующей метрике нелинейного дифференциального уравнения Монжа - Ампера. К таким геометрическим характеристикам относят гауссову кривизну, среднюю кривизну, сумму главных радиусов кривизны и др. В работе рассматриваются гомеоморфные сфере единичного радиуса поверхности из класса регулярных выпуклых в трехмерном пространстве постоянной отрицательной кривизны с заданной функцией внутренней (гауссовой) кривизны. Внутренняя кривизна рассматривается как функция точки трехмерного пространства Лобачевского. Решение дифференциального уравнения Монжа - Ампера предполагается функцией, заданной явно в сферических координатах. В работе изложена процедура построения априорных оценок первых производных решения уравнения. Предполагается наличие оценок самого решения.

Ключевые слюва: гиперболическое пространство; уравнение Монжа - Ампера; отрицательная эллиптичность; бельтрамиевы координаты; гауссова кривизна.

Для цитирования:

Филимонова А. П., Юрьева Т. А. Априорные оценки градиента решения уравнения некоторого класса Монжа - Ампера // Вестник Бурятского государственного университета. Математика, информатика. 2019. № 1. С. 49-55.

\section{Введение}

Рассмотрим вопрос восстановления выпуклой гомеоморфной сфере поверхности трехмерного пространства постоянной отрицательной кривизны (пространства Лобачевского, гиперболического пространства $H^{3}$ ) по ее внешней кривизне, заданной как функция точки пространства $H^{3}$.

Аналитически данная задача сводится к решению нелинейного уравнения с частными производными второго порядка (типа Монжа - Ампера) на сфере как двумерном многообразии. 


\section{1 Постановка задачи}

Поверхность считаем регулярной и звездной относительно некоторой фиксированной точки $O$ пространства $H^{3}$.

В $H^{3}$ фиксируется некоторая точка $O$ и сфера $S_{1}^{2}$ с центром в этой точке радиуса 1.

Регулярную выпуклую гомеоморфную $S_{1}^{2}$ и звездную относительно точки $O$ поверхность в сферических координатах $u, v, \rho$ можно задать уравнением $F: \rho=\rho(u, v)$.

Сформулированная выше геометрическая задача существования и единственности поверхности $F$ в пространстве $H^{3}$, гауссова кривизна которой в каждой точке равна значению заданной в $H^{3} \backslash\{O\}$ функции $K_{\text {int }}(u, v, \rho)=K_{i}$ в той же точке, сводится к исследованию следующего уравнения Монжа - Ампера $[1 ; 2]$ :

$$
\begin{aligned}
& \rho_{11} \rho_{22}-\rho_{12}^{2}-\rho_{11}\left(2 \operatorname{cth} \rho \cdot \rho_{v}^{2}+\operatorname{sh} \rho \cdot \operatorname{ch} \rho\right)+2 \rho_{12} \rho_{u} \rho_{v} \operatorname{cth} \rho- \\
& -\rho_{22}\left(2 \operatorname{cth} \rho \cdot \rho_{u}^{2}+\operatorname{sh} \rho \cdot \operatorname{ch} \rho \cos ^{2} v\right)- \\
& -\left(\rho_{v}^{2} \cos ^{2} v+\frac{\rho_{u}^{2}}{\cos v}\right)^{2}+2 \rho_{u}^{2}+2 \rho_{v}^{2} \cos ^{2} v+\operatorname{sh}^{2} \rho \cos ^{2} v= \\
& =K_{i}(u, v, \rho) \frac{\left(\rho_{u}^{2}+\rho_{v}^{2} \cos ^{2} v+\operatorname{sh}^{2} \rho \cdot \cos ^{2} v\right)^{2}}{\cos ^{2} v} .
\end{aligned}
$$

Здесь $\rho_{i j}$ - вторые ковариантные производные функции $\rho=\rho(u, v)$ относительно метрики единичной сферы $S_{1}^{2}$.

Условием отрицательной эллиптичности указанного выше уравнения является положительность внешней кривизны $K_{\text {ext }}=K_{\mathrm{int}}+1$.

При некоторых ограничениях на функцию $K_{i}(u, v, \rho)$ имеет место утверждение о расположении поверхности $F$, а именно: пусть в $H^{3}$ фиксированы две концентрические сферы $S_{\rho_{1}}^{2}$ и $S_{\rho_{2}}^{2}\left(\rho_{1}<\rho_{2}\right)$ с центром в точке $O$ и радиусами $\rho_{1}$ и $\rho_{2}$ соответственно. Пусть функция $K_{\mathrm{int}}(u, v, \rho)$, определенная в $S_{1}^{2} \times R^{+}$, удовлетворяет с условию: $K_{\text {int }}(u, v, \rho)>-1$ $\left(K_{\text {ext }}+1>0\right) ; \quad K_{i}(u, v, \rho)=\frac{1}{s h^{2} \rho}+h(u, v, \rho), \quad h>0$ внутри сферы $S_{\rho_{1}}^{2}$ и $h<0$ вне сферы $S_{\rho_{2}}^{2}$. Тогда любое решение $\rho=\rho(u, v)$ указанного выше уравнения Монжа - Ампера задает поверхность $F$, расположенную между сферами $S_{\rho_{1}}^{2}$ и $S_{\rho_{2}}^{2}[2]$.

Заметим, что атлас на многообразии $S_{1}^{2}$ выбран так, что в локальных координатах $u, v$ каждой карты $\cos v \geq \alpha>0$. 
А. П. Филимонова, Т. А. Юрьева. Априорные оценки градиента решения уравнения некоторого класса Монжа - Ампера

\section{2 Построение априорных оценок}

Рассмотрим процесс получения равномерных по $u, v$ априорных оценок первых производных решения $\rho_{u}(u, v)$ и $\rho_{v}(u, v)$ исследуемого уравнения.

$H^{3}$ геодезически отобразим в открытый единичный шар в евклидовом пространстве $E^{3}$ с центром в точке $O$ с декартовой системой координат $O, x, y, z$, то есть воспользуемся моделью Кэли - Клейна гиперболического пространства $H^{3}$. Координаты $x, y, z$ для точек $H^{3}$ называются бельтрамиевыми, линейный элемент пространства $H^{3}$ :

$$
d s^{2}=\frac{(x d x+y d y+z d z)^{2}+\left(1-x^{2}-y^{2}-z^{2}\right) \cdot\left(d x^{2}+d y^{2}+d z^{2}\right)}{\left(1-x^{2}-y^{2}-z^{2}\right)^{2}}[3] .
$$

Поверхность $F$ в этой модели изображается выпуклой евклидовой гомеоморфной сфере, точка $O$ лежит внутри $F$.

Пусть $M_{0}\left(u_{0}, v_{0}, \rho\left(u_{0}, v_{0}\right)\right)$ - фиксированная точка поверхности $F$, заданной функцией $\rho=\rho(u, v)$, где $\rho(u, v)$ - решение исследуемого уравнения Монжа - Ампера.

Рассуждения будем вести в карте $\gamma$ сферы $S_{1}^{2}$, где

$$
\gamma^{-1}:\left\{\begin{array}{l}
x=t h 1 \sin v, \\
y=t h 1 \cos v \sin u, \quad \cos v \geq \alpha>0 . \\
z=t h 1 \cos u \cos v,
\end{array}\right.
$$

В этом случае формулы перехода от сферических координат к бельтрамиевым принимают следующий вид:

$$
\left\{\begin{array}{l}
x=\operatorname{th} \rho \sin v \\
y=\operatorname{th} \rho \cos v \sin u \\
z=\operatorname{th} \rho \cos u \cos v
\end{array}\right.
$$

Рассмотрим движение $D$ пространства $H^{3}$, которое оставляет неподвижной точку $O(0,0,0)$ и переводит точку $M_{0}$ в точку $\bar{M}_{0}$, лежащую на отрицательной полуоси $O z$. Это движение можно задать матрицей:

$$
D=\left(\begin{array}{ccc}
\cos v_{0} & -\sin v_{0} \sin u_{0} & -\sin v_{0} \cos u_{0} \\
0 & \cos u_{0} & -\sin u_{0} \\
-\sin v_{0} & -\cos v_{0} \sin u_{0} & -\cos v_{0} \cos u_{0}
\end{array}\right) .
$$

В силу того, что поверхность $F$ расположена между сферами $S_{\rho_{1}}^{2}$ и $S_{\rho_{2}}^{2}$, то движение $D$ переводит поверхность $F$ в поверхность $\bar{F}=D(F)$, расположенную между сферами $D\left(S_{\rho_{1}}^{2}\right)=S_{\rho_{1}}^{2}$ и $D\left(S_{\rho_{2}}^{2}\right)=S_{\rho_{2}}^{2}$.

Возьмем на плоскости $x O y$ круг $K: x^{2}+y^{2}=t h^{2} \rho_{1}$ и вырежем прямым 
цилиндром с направляющей $K$ и образующей, параллельной оси $O z$ из $\bar{F}$ область, содержащую точку $\bar{M}_{0}$ и однозначно проектирующуюся на плоскость $x O y$. Эту область можно задать явным уравнением в бельтрамиевых координатах: $z=z(x, y)$.

Далее, рассмотрим круговой конус $K_{0}$ с вершиной в точке $\bar{M}_{0}$ и направляющей $K$. Этот конус обращен выпуклостью в сторону $z<0$, его вершина $\bar{M}_{0}$ имеет координаты $(0,0, z(0,0))$. Касательная плоскость к поверхности $z=z(x, y)$ в точке $\bar{M}_{0}$ является одной из опорных плоскостей конуса $K_{0}$. Нормальное изображение конуса $K_{0}$ представляет собой круг на плоскости $(p, q)$ с центром в точке $(0,0)$ [4]. Так как высота конуса не превосходит $t h \rho_{2}$, нормальное изображение конуса $K_{0}$ содержится в круге

$$
p^{2}+q^{2} \leq \frac{t h^{2} \rho_{2}}{t h^{2} \rho_{1}},
$$

следовательно,

$$
z_{x}^{2}(0,0)+z_{y}^{2}(0,0) \leq \frac{t h^{2} \rho_{2}}{t h^{2} \rho_{1}},
$$

отсюда имеем неравенства

$$
\left|z_{x}(0,0)\right| \leq \frac{t h \rho_{2}}{t h \rho_{1}} \text { и }\left|z_{y}(0,0)\right| \leq \frac{t h \rho_{2}}{t h \rho_{1}} .
$$

Теперь покажем, что из ограниченности первых производных функции $z=z(x, y)$ в точке $(0,0)$ следует ограниченность первых производных функции $\bar{\rho}(\bar{\rho}$ задает поверхность $\bar{F})$ в точке $(\pi, 0)$.

Точке $\bar{M}_{0}$ на $S_{1}^{2}$ соответствует точка $\left(\bar{u}_{0}, \bar{v}_{0}\right)=(\pi, 0)$, принадлежащая карте $\gamma$.

Так как $z_{u}=z_{x} \cdot x_{u}+z_{y} \cdot y_{u}, z_{v}=z_{x} \cdot x_{v}+z_{y} \cdot y_{v}$, то, используя формулы перехода от сферических координат к бельтрамиевым, будем иметь:

$$
\begin{gathered}
\bar{\rho}_{\bar{u}}\left(\cos \bar{v} \cos \bar{u}-z_{x} \cdot \sin \bar{v}-z_{y} \cos \bar{v} \sin \bar{u}\right)=\operatorname{sh} \bar{\rho} c h \bar{\rho} \cos \bar{v}\left(z_{x} \sin \bar{u}+z_{y} \cos \bar{u}\right), \\
\bar{\rho}_{\bar{v}}\left(\cos \bar{v} \cos \bar{u}-z_{x} \cdot \sin \bar{v}-z_{y} \cos \bar{v} \sin \bar{u}\right)= \\
=\operatorname{sh} \bar{\rho} \operatorname{ch} \bar{\rho}\left(\sin \bar{v} \cos \bar{u}+z_{x} \cos \bar{v}-z_{y} \sin \bar{v} \sin \bar{u}\right) .
\end{gathered}
$$

Тогда:

$$
\begin{gathered}
\bar{\rho}_{\bar{u}}(\pi, 0)=\operatorname{sh} \bar{\rho}(\pi, 0) \operatorname{ch} \bar{\rho}(\pi, 0) z_{y}(0,0), \\
\bar{\rho}_{\bar{v}}(\pi, 0)=-\operatorname{sh} \bar{\rho}(\pi, 0) \operatorname{ch} \bar{\rho}(\pi, 0) z_{x}(0,0) .
\end{gathered}
$$

Отсюда, используя имеющиеся выше результаты, будем иметь: 
А. П. Филимонова, Т. А. Юрьева. Априорные оценки градиента решения уравнения некоторого класса Монжа - Ампера

$$
\left|\bar{\rho}_{\bar{u}}^{-}(\pi, 0)\right| \leq \frac{s h^{2} \rho_{2}}{t h \rho_{1}},\left|\bar{\rho}_{\bar{v}}^{-}(\pi, 0)\right| \leq \frac{s h^{2} \rho_{2}}{t h \rho_{1}} .
$$

Далее, при движении $D$ образом произвольной точки $(x, y, z)$, где

$$
\left\{\begin{array}{l}
x=\operatorname{th} \rho \sin v \\
y=\operatorname{th} \rho \cos v \sin u \\
z=t h \rho \cos u \cos v
\end{array}\right.
$$

является точка $(\bar{x}, \bar{y}, \bar{z})$, где

$$
\left\{\begin{array}{l}
\bar{x}=\operatorname{th} \rho\left(\cos v_{0} \sin v-\sin v_{0} \cos v \cos \left(u-u_{0}\right)\right), \\
\bar{y}=\operatorname{th} \rho \cos v \sin \left(u-u_{0}\right), \\
\bar{z}=-t h \rho\left(\sin v_{0} \sin v+\cos v_{0} \cos v \cos \left(u-u_{0}\right)\right),
\end{array}\right.
$$

следовательно,

$\sin \bar{v}=\cos v_{0} \sin v-\sin v_{0} \cos v \cos \left(u-u_{0}\right), \cos \bar{v} \sin \bar{u}=\cos v \sin \left(u-u_{0}\right)$.

Продифференцируем последние равенства по $u$ и $v$ :

$$
\begin{gathered}
\cos \bar{v} \cdot \frac{\partial \bar{v}}{\partial u}=\sin v_{0} \cos v \sin \left(u-u_{0}\right) \\
\cos \bar{v} \cdot \frac{\partial \bar{v}}{\partial v}=\cos v_{0} \cos v+\sin v_{0} \sin v \cos \left(u-u_{0}\right) ; \\
\cos \bar{v} \cos \bar{u} \cdot \frac{\partial \bar{u}}{\partial u}-\sin \bar{v} \sin \bar{u} \cdot \frac{\partial \bar{v}}{\partial u}=\cos v \cos \left(u-u_{0}\right) ; \\
\cos \bar{v} \cos \bar{u} \cdot \frac{\partial \bar{u}}{\partial v}-\sin \bar{v} \sin \bar{u} \cdot \frac{\partial \bar{v}}{\partial v}=-\sin v \sin \left(u-u_{0}\right) .
\end{gathered}
$$

Отсюда следует, что

$$
\frac{\partial \bar{v}}{\partial u}\left(u_{0}, v_{0}\right)=0, \frac{\partial \bar{v}}{\partial v}\left(u_{0}, v_{0}\right)=1, \frac{\partial \bar{u}}{\partial u}\left(u_{0}, v_{0}\right)=-\cos v_{0}, \frac{\partial \bar{u}}{\partial v}\left(u_{0}, v_{0}\right)=0
$$

Тогда

$$
\begin{gathered}
\rho_{u}\left(u_{0}, v_{0}\right)=\bar{\rho}_{\bar{u}}(\pi, 0) \frac{\partial \bar{u}}{\partial u}\left(u_{0}, v_{0}\right)+\bar{\rho}_{\bar{v}}^{-}(\pi, 0) \frac{\partial \bar{v}}{\partial u}\left(u_{0}, v_{0}\right)=-\bar{\rho}_{\bar{u}}(\pi, 0) \cos v_{0} ; \\
\rho_{v}\left(u_{0}, v_{0}\right)=\bar{\rho}_{\bar{u}}^{-}(\pi, 0) \frac{\partial \bar{u}}{\partial v}\left(u_{0}, v_{0}\right)+\bar{\rho}_{\bar{v}}^{-}(\pi, 0) \frac{\partial \bar{v}}{\partial v}\left(u_{0}, v_{0}\right)=-\bar{\rho}_{\bar{v}}^{-}(\pi, 0) .
\end{gathered}
$$

Отсюда и из предыдущих выкладок имеем:

$$
\left|\rho_{u}\left(u_{0}, v_{0}\right)\right| \leq \frac{s h^{2} \rho_{2}}{\operatorname{th} \rho_{1}},\left|\rho_{v}\left(u_{0}, v_{0}\right)\right| \leq \frac{s h^{2} \rho_{2}}{\operatorname{th} \rho_{1}} .
$$

Если точка сферы принадлежит области определения другой ее карты $\gamma_{1}$, то, записывая для $\gamma_{1}$ формулы перехода от сферических координат к бельтрамиевым, выбираем движение $D$ так, чтобы оно оставляло $O(0,0,0)$ неподвижной, а нашу точку переводило на определяемую по $\gamma_{1}$ 
координатную полуось. Направляющую $K$ цилиндра проектирования берем на ортогональной этой полуоси координатной плоскости.

Все остальные рассуждения полностью аналогичны приведенным выше для карты $\gamma$.

\section{Заключение}

Для любой точки сферы $S_{1}{ }_{1}$ имеем априорные оценки первых производных функции $\rho=\rho(u, v)$, которая является решением исследуемого уравнения Монжа - Ампера.

А именно, имеет место следующий результат: в условиях ограничений на функцию $K_{\text {int }}=K_{i}(u, v, \rho)$, сформулированных ранее, есть априорная оценка первых производных функции $\rho=\rho(u, v)$ (то есть градиента решения уравнения Монжа - Ампера):

$$
\left|\rho_{u}\left(u_{0}, v_{0}\right)\right| \leq \frac{s h^{2} \rho_{2}}{\operatorname{th} \rho_{1}},\left|\rho_{v}\left(u_{0}, v_{0}\right)\right| \leq \frac{s h^{2} \rho_{2}}{\operatorname{th} \rho_{1}} .
$$

\section{Литература}

1. Филимонова А. П., Юрьева Т. А. Аналог теорем расположения замкнутых выпуклых поверхностей с заданной функцией внутренней кривизны в пространствах постоянной кривизны // Вестник АмГУ. 2017. Вып. 79. С. 17-21.

2. Филимонова А. П., Юрьева Т. А. Априорные оценки решения в метрике $C^{0}\left(S_{1}^{2}\right)$ уравнения типа Монжа - Ампера на сфере как двумерном многообразии в пространстве постоянной кривизны // Международный научноисследовательский журнал. 2016. № 9-2(51). С. 132-136.

3. Филимонова А. П., Юрьева Т. А. Свойство выпуклости функции внешней кривизны поверхности в трехмерном пространстве Лобачевского // Вестник АмГУ. 2015. Вып. 69. С. 22-25.

4. Погорелов А. В. Многомерное уравнение Монжа - Ампера. М.: Наука, 1988. $96 \mathrm{c}$.

\section{PRIOR ESTIMATES OF THE GRADIENT FOR SOLUTION OF A CERTAIN}

MONGE-AMPÈRE EQUATION

Anna P. Filimonova

Cand. Sci. (Phys. and Math.), A/Prof.,

Amur State University,

21 Ignatyevskoe Highway, Blagoveschensk 675000, Russia

E-mail: ap.filimonova@mail.ru

Tatyana A. Yuryeva

Cand. Sci. (Education), A/Prof.,

Amur State University,

21 Ignatievskoe Highway, Blagoveschensk 675000, Russia

E-mail: yuryevatat@mail.ru 
А. П. Филимонова, Т. А. Юрьева. Априорные оценки градиента решения уравнения некоторого класса Монжа - Ампера

The resolution of the issue of existence and uniqueness of surfaces with given geometric characteristics in various spaces is associated with finding prior estimates for solution of a nonlinear Monge-Ampère differential equation in the corresponding metrics. Such geometric characteristics include Gaussian curvature, average curvature, the sum of principal radii of curvature, etc. The article describes surfaces homeomorphic to the sphere of a unit radius from the class of regular convex in threedimensional space of constant negative curvature with a given function of intrinsic (Gaussian) curvature. Intrinsic curvature is considered as a function of the point of three-dimensional Lobachevsky space. The solution for a Monge-Ampère differential equation is assumed to be a function explicitly given in spherical coordinates. The article describes the procedure for constructing prior estimates of the first derivatives of equation solution. It is assumed the availability of estimates of the solution itself.

Keywords: hyperbolic space; Monge-Ampère equation; negative ellipticity; Beltrami coordinates; Gaussian curvature.

\section{References}

1. Filimonova A. P., Yuryeva T. A. Analog teorem raspolozheniya zamknutykh vypuklykh poverkhnostei s zadannoi funktsiei vnutrennei krivizny $v$ prostranstvakh postoyannoi krivizny [Analogue of Theorems for the Location of Closed Convex Surfaces with a Prescribed Function of Intrinsic Curvature in Constant Curvature Spaces]. Vestnik Amurskogo gosudarstvennogo universiteta. 2017. V. 79. Pp. 17-21.

2. Filimonova A. P., Yuryeva T. A. Apriornye otsenki resheniya v metrike uravneniya tipa Monzha-Ampera na sfere kak dvumernom mnogoobrazii v prostranstve postoyannoi krivizny [Prior Estimates of the Solution of a Monge-Ampére Type Equation in $C^{0}\left(S_{1}^{2}\right)$ Metrics on a Sphere as Two-Dimensional Manifold in Constant Curvature Space]. Mezhdunarodnyi nauchno-issledovatelskii zhurnal. 2016. No. 92(51). Pp. 132-136.

3. Filimonova A. P., Yuryeva T. A. Svoistvo vypuklosti funktsii vneshnei krivizny poverkhnosti v trekhmernom prostranstve Lobachevskogo [A Convexity Property of the Function of Surface External Curvature in Three-Dimensional Lobachevsky Space]. Vestnik Amurskogo gosudarstvennogo universiteta. 2015. V. 69. Pp. 22-25.

4. Pogorelov A. V. Mnogomernoe uravnenie Monzha-Ampera [Multidimensional Monge-Ampère Equation]. Moscow: Nauka Publ., 1988. 96 p. 